\title{
Fine needle aspiration cytology findings of primary pulmonary choriocarcinoma in a male patient
}

\begin{abstract}
Here we are presenting a case of a 26-year-old male who presented with respiratory distress and constitutional symptoms. CT-guided FNAC was performed and the cytomorphological features were suggestive of a non-small cell carcinoma with extensive necrosis. However, being a non-smoker and presence of multiple cannonball lesions in the lungs, young age of the patient and markedly elevated serum $\beta$-HCG level raised the suspicion of a germ cell tumor. The testicles and other relevant organs were clinically and radiologically normal. This aroused the possibility of primary pulmonary choriocarcinoma and eventually the diagnosis was confirmed by biopsy and immunohistochemistry.
\end{abstract}

Keywords: primary pulmonary choriocarcinoma, FNAC, squamous cell carcinoma, non-seminomatous germ cell tumor, $\beta$ - HCG
Volume 2 Issue 3 - 2017

\author{
Swapan Samanta' Nirmal Sultania ${ }^{2}$ \\ 'Probe diagnostic Pvt Ltd, India \\ ${ }^{2} E K O$ Diagnostic Pvt Ltd, India
}

Correspondence: Swapan Samanta, Probe diagnostic Pvt Ltd, I2I,AJC Bose Road, Kolkata-7000I4, India, Tel 9733365004 , Email swapansmnt@gmail.com

Received: November 10, 2016 | Published: May 22, 2017

\section{Case report}

Extragonadal germ cell tumors (EGCT) account for 2-5\% of all germ cell tumors. ${ }^{1}$ Choriocarcinoma in male subjects is one of the non-seminomatous EGCT that occurs in relatively young individuals. Primary (extragonadal) choriocarcinoma (PCC) commonly arises in the retro peritoneum, mediastinum and brain. ${ }^{2}$ Metastasis to the lung is common, but PCC originating in the lung is extremely rare. Cytological features of PCC have been described only occasionally., Here we describe a case of a 26 years old male where the differential diagnosis of PCC was offered after FNAC (fine needle aspiration cytology) and subsequently it was confirmed histologically and immunohistochemically. The prognosis of pulmonary PCC, especially in males is very poor as compared to its testicular counterpart because hematogenous spread is usually common at the time of diagnosis..$^{2,5,6}$ Often the entity is misdiagnosed as more common diseases such as primary or secondary lung tumors, tuberculosis, pneumonia or lymphoma and thus potentially curative chemotherapy or surgery may be delayed..$^{5-7}$ Considering its rarity, prognosis and diagnostic difficulties FNAC definitely has a role in such cases! A 26 year-old non-smoker male with no significant medical or surgical history presented with the complaints of progressive shortness of breath, productive cough, night sweats, diminished appetite and unexplained weight loss for 4 weeks. Sputum for AFB (Acid-Fast Bacilli) was negative.

Mantoux test was negative. No history of hemoptysis, chest pain, fever or chills was present. The patient did not respond to routine antibiotics. Chest examination revealed diffuse coarse inspiratory crackles. Genital exam did not reveal any scrotal mass. Laboratory data revealed raised ESR, neutrophilic leucocytosis, normal liver function tests and alpha-fetoprotein level. HIV 1 and 2 were nonreactive. Chest x-ray showed bilateral pulmonary cannon-ball nodules. Computed tomography (CT) demonstrated multiple cannonball lesions of variable sizes in both lung fields largest one measuring $8 \times 7 \times 5 \mathrm{~cm}$ without enlargement of intrapulmonary or mediastinal lymph nodes (Figure 1A) that first raised the suspicion of a germ cell tumor.

FNAC under CT-guidance (Figure 1B) was suggestive of nonsmall cell carcinoma possibly squamous cell carcinoma with extensive necrosis (Figures 1C \& 1D). The cellularity was poor and the fields were predominated by hemorrhage and large areas of coagulative necrosis and ghost cells resembling anucleate squames (Figures 1C \& 1D). Many apoptotic and degenerated cells and cells with cytoplasmic orangeophilia were identified (Figure 1d) but practically no inflammatory cells! The tumor cells were large, undifferentiated, highly pleomorphic with clumped chromatin, indistinct nucleoli and multivacuolated abundant cytoplasm (Figures 2A-2C). Bi and multinucleated cells were present. Screening for additional mass including CT scan of the retroperitoneum, abdomen, scrotum, brain and spine showed no abnormality. Serum levels of Human chorionic gonadotrophin $(\beta-\mathrm{HCG})$ and lactate de hydrogenase which were elevated $(40240 \mathrm{mIU} / \mathrm{ml}$ and $870 \mathrm{IU} / \mathrm{dl}$ respectively). CT-guided coreneedle biopsy (Figure 2D) of the left lung mass showed large necrotic and hemorrhagic areas with lamellae of large polygonal multinucleated/ bizarre cells representing syncytiotrophoblast punctuated by medium to small-sized cytotrophoblasts with clear cytoplasm characteristic of choriocarcinoma (Figure 2D). Immunohisto-chemistry showed positivity for cytokeratin, $\beta-\mathrm{HCG}$ and negativity for PLAP, CD-30 and alpha feto-protein. The diagnosis of PPC was confirmed. The patient received five cycles of $20 \mathrm{mg} / \mathrm{m} 2$ of cisplatin, $100 \mathrm{mg} / \mathrm{m} 2$ of etoposide and 30 units of bleomycin as per standard protocol. Supportive care was provided as well. The patient has improved markedly and is being monitored with serial serum $\beta$-HCG levels that dropped from $40240 \mathrm{mIU} / \mathrm{ml}$ to $20 \mathrm{mIU} / \mathrm{ml}$.

The patient, after 8 months of diagnosis is doing well. Choriocarcinoma most commonly occurs in the female genital tract following gestational events such as hydatidiform mole, normal pregnancy, abortion and ectopic pregnancy. In men, choriocarcinoma occurs mostly in the testes. ${ }^{1}$ PCC of the lung is extremely rare. The tumor also occurs in females in the absence of pregnancy and also in males. ${ }^{1,2,5}$ Primary EGCT is rare and usually presents as a midline lesion in the retroperitoneum, mediastinum, or cranial cavity. Less frequently, these tumors have also been reported in other organs such as the urinary bladder, kidney, liver, stomach and colon. Rather, lung is a common site of metastatic choriocarcinoma. ${ }^{1,2,-7}$ Preoperative diagnosis of PCC of the lung is difficult. So, a careful search for an occult primary (elsewhere) is necessary. Not only immunohistochemical studies but serum levels of HCG have 
also been approved for the detection of PCC.$^{8-10}$ Here, FNAC was performed and cytomorphology was suggestive squamous cell carcinoma, based on the clumps of ghost cells/apoptotic cells and necrosis. Other possibilities were undifferentiated malignant tumor, large cell carcinoma, adenosquamous carcinoma, giant cell-rich or metaplastic carcinoma, anaplastic carcinoma, and pleomorphic undifferentiated sarcoma etc. Still high $\beta$-HCG level and cannon ball deposits could not be explained. Other organs especially the retroperitoneum were evaluated which excluded any tumor. Because elevated HGC is associated with choriocarcinoma, so the possibility of PCC was suggested. Confirmation was done by biopsy (Figure 2D) and immunohistochemistry. Urgently chemotherapy was started and the patient responded well to the standard protocol. Prognosis of PPC according to literature is worse than its testicular counterpart ${ }^{5-7}$ but the present patient is doing relatively well possibly because the tumor is restricted to the lungs only.

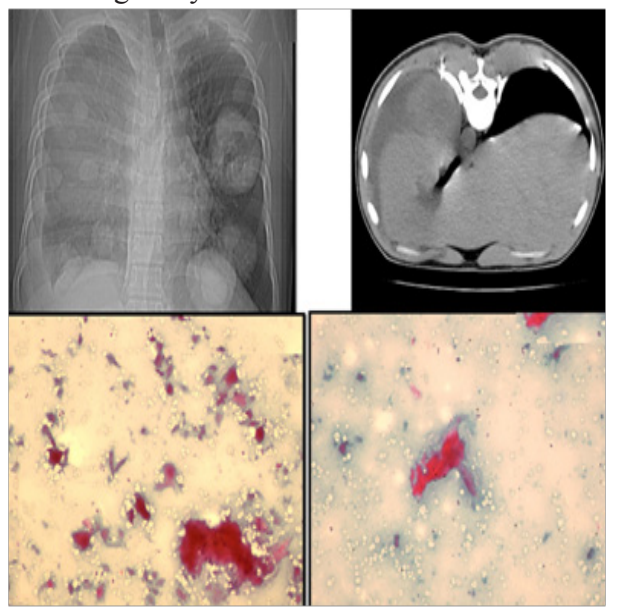

Figure I Clock-wise from upper left: CT scan revealed cannon-ball lesions in both lung fields largest one measuring $8 \times 7 \times 5 \mathrm{~cm}$ without enlargement of lymph nodes. FNAC done under CT-guidance (needle shown In-situ).The cytosmears: cellularity was poor; the fields were predominated by hemorrhage/coagulative necrosis and ghost cells resembling anucleate squames. Many apoptotic and degenerated cells and few with orangophilic cytoplasm but virtually no inflammatory cells (PAP I00X)

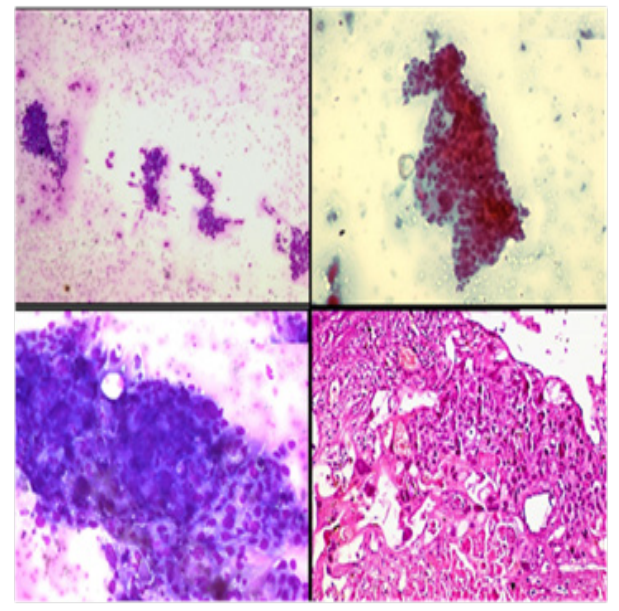

Figure 2 Upper two (MGG 40X; PAP I00X) and lower left phomicrograph (MGG I00X): Details of cytomorphology of the tumor: the tumor cells were large, undifferentiated, highly pleomorphic, with clumping of chromatin and indistinct nucleoli and multivacuolated cytoplasm. Binucleate and multinucleate cells were present. Lower right photomicrograph: CT-guided trans-thoracic needle biopsy confirmed choriocarcinoma (H \& E, I00X).
Cytomorphology of PCC parallels that of an undifferentiated highly malignant tumor. However, cases presents with extensive necrosis, hemorrhage and ghost tumor cells resembling anucleate squames should raise the suspicion thus cytology and clinical correlation with $\beta$-HCG level may help in rapid diagnosis. The cytological features of choriocarcinoma are quite characteristic as described in the present article. These cases may easily be misdiagnosed as common malignancies like squamous cell carcinoma.

\section{Acknowledgements}

Dr. S. K. Sharma, Chief radiologist and director, EKO Diagnostic Pvt. Ltd. 54, Jawaharlal Nehru Road, Kolkata-700071.

\section{Conflict of interest}

The author declares no conflict of interest.

\section{References}

1. Collins DH, Pugh RC. Classification and frequency of testicular tumours. Br J Urol. 1964;36(Suppl):1-11.

2. Bokemeyer C, Nichols CR, Droz JP. Extragonadal germ cell tumors of the mediastinum and retroperitoneum: results from an international analysis. J Clin Oncol. 2002;20(7):1864-1873.

3. Saha K, Basuthakur S, Jash D, et al. Gestational choriocarcinoma presenting as hemothorax. Indian J Med Sci. 2010;64(5):237-240.

4. Choi HJ, Park IA. Fine needle aspiration cytology of metastatic choriocarcinoma presenting as a breast lump. A case report. Acta Cytol. 2004;48(1):91-94.

5. Kuang XW. Primary pulmonary choriocarcinoma in a male: report of a case and review of the literature. Zhonghua Jie He He Hu Xi Xi Ji Bing Za Zhi. 1984;7(6):354-355.

6. Sridhar KS, Saldana MJ, Thurer RJ, et al. Primary choriocarcinoma of the lung: report of a case treated with intensive multimodality therapy and review of the literature. J Surg Oncol. 1989;41(2):93-97.

7. Fine G, Smith RW, Pachter MR. Primary extragenital choriocarcinoma in the male subject. Case report and review of the literature. Am J Med. 1962;32:776-794.

8. Toda S, Inoue $\mathrm{Y}$, Ishino $\mathrm{T}$, et al. A rare case of primary pulmonary choriocarcinoma in a male: immunohistochemical detection for human chorionic gonadotrophin, epidermal growth factor $(\mathrm{EGF})$ and ECF-receptor. Endocr J. 1995;42(5):655-659.

9. Ikura Y, Inoue T, Tsukuda H. Primary choriocarcinoma and human chorionic gonadotrophin-producing giant cell carcinoma of the lung: are they independent entities. Histopathology. 2000;36(1):17-25.

10. Tsai JR, Chong IW, Hung JY, et al. Use of urine pregnancy test for rapid diagnosis of primary pulmonary choriocarcinoma in a man. Chest. 2002;121(3):996-998. 